\title{
Introduction to special issue on noise modeling
}

\author{
Tomas Gonzalez
}

Published online: 21 February 2015

(c) Springer Science+Business Media New York 2015

Electronic noise is a limiting factor in many applications of semiconductor devices and integrated circuits. The fluctuations of electronic signals around their average (or cyclostationary) value can make impossible the recognition of the signal being processed or detected, which remains hidden by the noise generated by the device or the circuit. This can be particularly critical when working with low-power signals, like in many applications related to communications or remote sensing.

Fluctuations reflect the presence of the many processes taking place at a microscopic level and having an influence on macroscopic quantities like voltages or currents. The exact knowledge of such microscopic processes at the origin of fluctuations, and how they are transferred to the terminals of the devices, and eventually amplified or frequency-shifted in a given circuit, is essential for the control of the noise level in advanced electronic systems. Thus, efforts at material, device and circuit levels are necessary.

Different types of noise sources must be considered to cover the full scenario when going from low to high frequency: the thermal noise inherent to the materials, transformed into hot-carrier noise when operating under far from equilibrium conditions; the ubiquitous $1 / \mathrm{f}$ noise, described by theories based on mobility and/or number fluctuations; generation-recombination (GR) noise originated by carrier trapping, with cutoff frequencies related to the carrier lifetime; shot noise associated to carrier granularity; etc. Additionally, correlation between noise sources must be taken into account. Processes of noise upconversion originated by the nonlinear response of devices and circuits must be also

T. Gonzalez ( $\triangleleft)$

Departamento de Fisica Aplicada, Facultad de Ciencias, Universidad de Salamanca, Plaza de la Merced s/n, 37008 Salamanca, Spain

e-mail: tomasg@usal.es carefully considered, since they can be crucial in the performance of nonlinear circuits like oscillators and their associated phase noise.

In this context, a correct modelling of noise in devices and circuits becomes crucial for the development and progress of any electronic technology. Computational models accounting for all the aforementioned noise sources are needed for the design of appropriate strategies in order to control and/or reduce the noise present in a given device/system. At this point, modelling techniques at different physical levels are necessary. Technology Computed Aided Design (TCAD) tools for circuit analysis rely on compact noise models, based whether on empirical fittings of experimental data or on expressions obtained from the device physics. In the case of device simulation, Langevin approaches, consisting in adding a force describing the source of fluctuations to the equations of the device transport model (going from the Boltzmann equation to higher level hydrodynamic or driftdiffusion equations) are commonly used. The noise analysis by means of time-domain techniques based on carrier trajectories (whether semi-classical or quantum), like the Monte Carlo method, even if less computationally efficient, provide quite useful information about the physical processes taking place inside the devices at the origin of the noise behavior. They are especially useful for the analysis of high-frequency electronic devices, operating in the $\mathrm{GHz}-\mathrm{THz}$ ranges, while presenting an inherent difficulty to include processes with significantly different time-scales.

However, noise is not only an obstacle to signal detection and amplification. The level of noise and its frequency dependence can provide very useful information about carrier kinetics of electrons inside the devices, no longer available through the knowledge of the DC behavior. Typically, such is the case of mesoscopic systems, where the level of shot-noise suppression or enhancement is strongly linked 
to the transport regime inside the devices. Here modelling techniques, mostly based on quantum approaches, become also essential to correctly predict the noise behavior of the systems according to the dominating transport processes. Coulomb repulsion and Pauli principle (and the associated Fermi statistics) are essential ingredients in these models.

This Special Issue on Noise Modelling collects several invited and contributed papers covering a broad range of the topics previously mentioned and providing an overview of the state-of-the-art in noise modelling. They illustrate the hierarchy of the different techniques to be used depending on the system under analysis and the required accuracy. The complexity of the models increases as they move deeper into the physics of the microscopic processes at the origin of noise.

The first two papers are devoted to the modelling of lowfrequency (LF) noise processes. The paper by Nallatamby et al. analyzes GR noise in InGaP-GaAs HBTs. They combine a TCAD simulator (mainly used to calculate Green functions) with GR Langevin sources (whose parameters are obtained from LF noise measurements) to predict the associated noise at the terminals of HBTs, achieving a remarkable agreement between simulations and experimental results. The paper of Both et al. illustrates how to compute 1/f noise in MOSFETs operating under cyclo-stationary (CS) conditions by modifying the parameters of flicker noise used by BSIM4 MOSFET model. The modification is based on previous transient simulations in which the average Fermi level in the MOSFET channel is calculated and on the assumption of U-shape distribution of traps. The simulations, thus accounting for the influence of trap activity under CS conditions, predict correctly the reduction of the $1 /$ f power spectral density observed in the CS operation of MOSFETs.

Continuing with noise in MOSFETs, in their paper Ruic and Jungemann show that the analysis of nanoscale devices requires the self-consistent solution of Boltzmann, Schrödinger and Poisson equations and present a deterministic solver. They provide information about mathematical and numerical aspects of the implementation. By including a Langevin source in Boltzmann equation, and via Green's functions, they are able to analyze the noise behavior of the transistors. Detailed and accurate results on smallsignal and noise performance of nanoscale MOSFETs can be obtained with this type of approach. The Langevin approach is also used for noise calculations in the papers of Jungemann, dealing with noise in organic semiconductor devices, and Ramonas and Jungemann, on non-equilibrium electronphonon systems.

Noise is the ultimate limitation in the performance of oscillators, where phase noise is at the origin of frequency fluctuations. Traversa et al. review in their paper state decomposition techniques for the analysis of noise properties of autonomous oscillators, allowing the simultaneous assessment of phase and amplitude noise terms.

Three papers are devoted to the analysis of noise in highfrequency electronic devices. The paper by Sakalas et al. deals with compact modelling of noise in SiGe and InP HBTs in a broad frequency range reaching $\mathrm{mm}$-waves. Correlated base and collector noise sources are considered in the model, which is validated by comparison with measurements and simulations based on hydrodynamic and Boltzmann-Poisson equations. SiGe and InP technologies are compared in terms of minimum noise figure (NFmin). SiGe HBTs are found to exhibit lower NFmin, even if InP transistors become competitive beyond $100 \mathrm{GHz}$.

Monte Carlo (MC) simulation of high-frequency noise in advanced III-V HEMTs is the topic of the paper by Mateos et al. The authors detail the techniques used for the analysis of small-signal and noise behavior of transistors by means of MC simulations. Results on InGaAs and InAs technologies are reported. In order to correctly reproduce experimental results, different real effects have been included in MC simulators, like the influence of degeneracy, dielectrics, surface charges and contact parasitics. Noise associated to kink effect originated by impact ionization is also studied. The simulation results when varying different technological parameters are used to provide design rules for the low-noise optimization of the transistors when scaling down the dimensions in order to reach operation frequencies above $100 \mathrm{GHz}$.

Noise in field effect transistors used as $\mathrm{THz}$ detectors is the subject of the paper by Palermo et al. They use the hydrodynamic-Langevin approach to simulate noise in an InGaAs HEMT, where they observe the excitation of 2Dplasma waves. Such plasma waves originate a series of resonant peaks in the noise spectral densities of currents at the terminals, whose presence can be controlled by the embedding circuit. High-frequency excess noise is found to be damped by photo-excitation with beating frequencies in the $\mathrm{THz}$ range. Channel ungated regions are found to play a key role on the noise found around the plasma-wave frequencies.

Finally, three papers deal with noise modelling in systems where quantum transport models must be used for their correct analysis. Shot noise suppression and enhancement accounting for Colulomb interaction in one-dimensional field effect transistors is analyzed in the paper by Iannaccone et al. Carbon nanotube (CNT) and silicon nanowire (SNW) FETs are studied. In order to include the effect of Coulomb interaction, self-consistent simulations of the 3D Poisson equation coupled with the Schrödinger equation, within the non-equilibrium Green's function formalism, are performed. Quantum ballistic transport is assumed. To model the stochastic injection of electrons from the reservoirs, a MC technique is used to determine the occupation of the different channels, obeying Fermi-Dirac statistics. The authors show that by using Landauer-Büttiker formula, which neglects 
the influence of Coulomb interaction, noise can be overestimated.

The paper by Maccuci et al. is also devoted to shot noise suppression, in this case in disordered conductors subjected to magnetic fields. The numerical method used for the calculations is based on the recursive Green's function approach. 2D conductors with randomly distributed scatterers are considered. The results are discussed in terms of the interplay between the different characteristic lengths of the system: mean free path, localization length, separation between scatterers and cyclotron radius. Starting from a diffusive or quasidiffusive behavior, shot noise is increasingly suppressed as the magnetic field is turned on due to the reduction of backscattering.

The paper of Marian et al. is devoted to the computation of quantum noise by means of a simulator based on Bohmian trajectories. Even if the approach is fully quantum, since the technique is based on trajectories, the noise calculation is performed similarly as in semiclassical MC simulations. The sources of randomness in this approach are the initial con- ditions of simulated electrons (energy, momentum, injection time) and the initial position in the quantum trajectories, which is selected randomly according to the conditional wave function. Results on low and high frequency behavior of shot noise in a resonant tunneling diode are obtained and explained to illustrate the usefulness of the approach. An interesting discussion on the interpretation, computation and measurability of quantum noise is also included in the paper.

The research on noise modelling is evolving continuously, like computational electronics itself. Novel models and techniques are emerging that can be extended to the calculation of noise. Multiscale and multiphysics approaches are more and more used to analyze a diversity of electronic systems, and they can be also quite useful for noise modelling. Quantum simulators should shorten the distance between too theoretical/formal problems and the real noise issues in nanoscale transistors, blurred by the reduction of dimensions. The influence of thermal effects and variability issues on the noise performance of nanoscale electronic devices should also receive increased attention. 